\title{
Trinucleon Electric Dipole Moments in Chiral EFT
}

\author{
Alex Gnech* \\ Gran Sasso Science Institute, I-67100 L'Aquila, Italy, \\ Istituto Nazionale di Fisica Nucleare, sezione di Pisa, I-56100 Pisa, Italy \\ E-mail: alex.gnechegsi.it \\ Michele Viviani \\ Istituto Nazionale di Fisica Nucleare, sezione di Pisa, I-56100 Pisa, Italy \\ E-mail: michele.viviani@pi.infn.it
}

\begin{abstract}
Time reversal violation (TRV) sources in fundamental theories induce interactions between nucleons which can be revealed by looking at the presence of permanent electric dipole moments (EDM) of light nuclei. By using the chiral effective filed theory ( $\chi$ EFT) it is possible to connect the nuclear observables to the fundamental terms which induce TRV. In this work we derive the TRV nucleon-nucleon and three-nucleon potential up to next-to-next-to leading order (N2LO) by using $\chi$ EFT. The TRV interaction is then used to evaluate the EDM of ${ }^{3} \mathrm{H}$ and ${ }^{3} \mathrm{He}$ focusing in particular on the calculation of the theoretical errors and the convergence of the chiral expansion.
\end{abstract}

The 9th International workshop on Chiral Dynamics

17-21 September 2018

Durham, NC, USA

${ }^{*}$ Speaker. 


\section{Introduction}

Time Reversal Violation (TRV) and Parity Violation (PV) are key ingredients in the explanation of the observed baryon-antibaryon asymmetry in the Universe (BAU) [1]. The Standard Model (SM) has a natural source of CP-violation in the Cabibbo-Kobayashi- Maskawa (CKM) quark mixing matrix, however this mechanism is not sufficient to explain the observed value of BAU [2]. This discrepancy opens a window in possible TRV effect in extension of SM, such as the $\theta$-term in the Quantum Chromodynamics (QCD) sector [3], or in beyond-SM (BSM) theories [4].

The measurement of Electric Dipole Moments (EDMs) of particles is the most promising observable for studying TRV effects beyond CKM mixing matrix. The present experimental upper bounds on the EDMs are $\left|d_{n}\right|<2.9 \cdot 10^{-13} e$ fm for neutron [5], $\left|d_{p}\right|<7.9 \cdot 10^{-12} e$ fm for proton [6,7], and $\left|d_{e}\right|<8.7 \cdot 10^{-16} e$ fm for electron [8]. In this context, there are proposals of direct measurements of EDMs of charged particles in dedicated storage rings [9-13]. However, a single measurement would not be sufficient to identify the source of TRV. For this reason, the measurement of EDMs of various light nuclei such as ${ }^{2} \mathrm{H},{ }^{3} \mathrm{H}$ and ${ }^{3} \mathrm{He}$ can help to impose constrains on the TRV sources.

The use of light nuclei as probes for TRV results to be advantageous because the nuclear physics of the systems is theoretically under control. In particular, the chiral effective field theory $(\chi$ EFT) provides a systematic and perturbative scheme to study TRV nuclear effects treating all the possible sources [14-16]. Each Lagrangian term is associated to a low-energy constants (LECs) which must be determined fitting the experimental data. The $\chi$ EFT approach permits not only to determine the TRV interactions but it provides also a systematic way to control the errors due to the truncation of the chiral expansion [21].

In this work, starting from the Lagrangian of Refs. [14-16] adding only the isotensor interactions we derived the chiral potential up to next-to-next-leading order (N2LO). The potential is then used to study the EDM of the three nucleon system, ${ }^{3} \mathrm{H}$ and ${ }^{3} \mathrm{He}$, focusing in particular on the role of TRV three-body forces and in the estimation of the theoretical uncertainties. Such an approach provides a suitable framework for the future determination of the LECs.

\section{The nuclear TRV potential}

The relevant terms of the Lagrangian which can give contribution to the nuclear potential up to N2LO are [14-16],

$$
\begin{aligned}
\mathscr{L}_{\mathrm{TRV}}= & g_{0} \bar{\psi} \vec{\pi} \cdot \vec{\tau} \psi+g_{1} \bar{\psi} \pi_{3} \psi+g_{2} \bar{\psi} \pi_{3} \tau_{3} \psi+M \Delta \pi_{3} \pi^{2} \\
& -2 i \bar{\psi}\left(d_{0}+d_{1} \tau_{z}\right) \gamma_{5} \sigma^{\mu v} \psi F_{\mu \nu}+\mathscr{L}_{\mathrm{TRV}}^{N N},
\end{aligned}
$$

where $\mathscr{L}_{\mathrm{TRV}}^{N N}$ includes five contact interactions which permits us to take care of the exchange of heavier mesons and reabsorb the divergences in the potential. Further terms of the Lagrangian involving derivatives would give rise to potential terms which can be reabsorbed in those generated by the Lagrangian given in Eq. (2.1) [17]. Moreover, the calculation of the EDM requires the inclusion of the electromagnetic currents,

$$
\mathscr{L}_{\mathrm{TRV}}^{\gamma}=-2 \bar{\psi}\left(d_{0}+d_{1} \tau_{3}\right) \gamma_{5} \sigma^{\mu v} \psi F_{\mu v},
$$


from which we can define $d_{p}=\left(d_{0}+d_{1}\right) / 2$ and $d_{n}=\left(d_{0}-d_{1}\right) / 2$ which are the proton and neutron proper EDM.

We evaluate the $T$ matrix in terms of time-ordered perturbation theory amplitudes whose associated diagrams are shown in Figure 1. The nuclear potential was then derived from the $T$ matrix by inverting order by order in the power counting the Lippman-Schwinger equation. The leading order

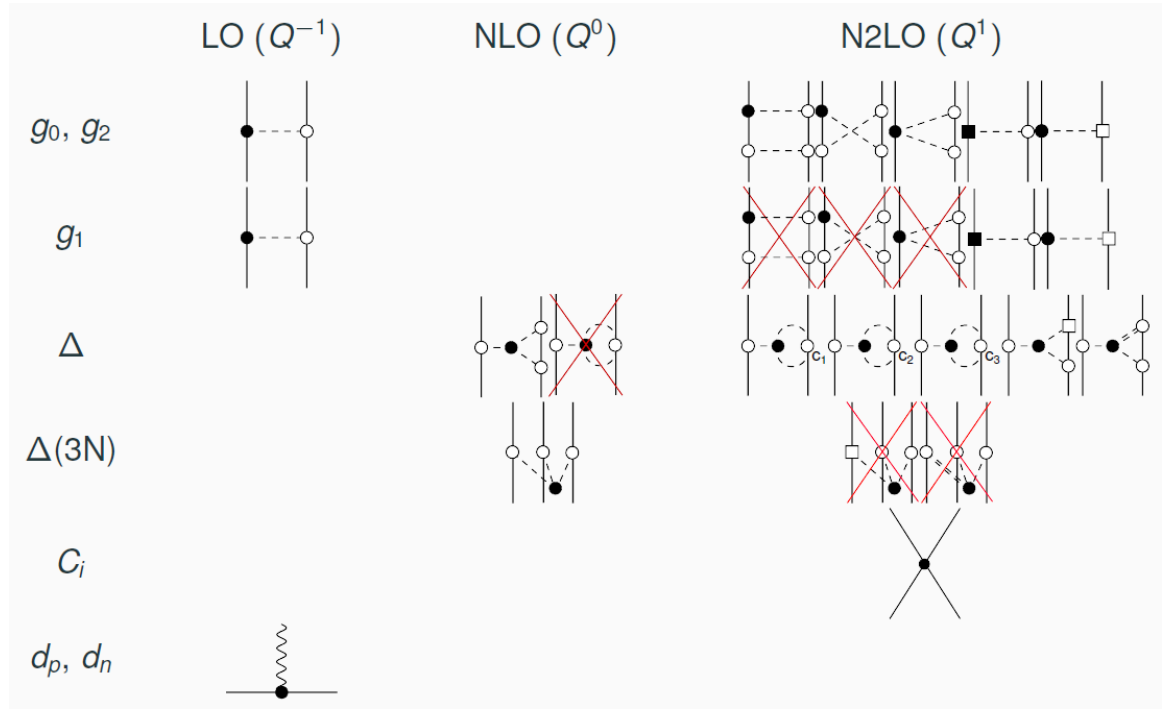

Figure 1: Time-ordered diagrams contributing to the TRV potential. Nucleons and pions are denoted by solid and dashed lines, respectively. The open (solid) circle represents a PC (TRV) vertex. The squares represent NLO vertices, while the double dashed lines recoil corrections coming from the energy denominators.

(LO) of the potential is given by the one pion exchange (OPE) associated to the LECs $g_{0}, g_{1}$ and $g_{2}$. The two pion exchange (TPE) contribution coming from these LECs appears only for $g_{0}$ and $g_{2}$ as already observed in Ref. [18]. The three pion vertex (TPV) gives rise to contribution at order $Q^{0}$ but also of order $Q^{1}$ when the LECs $c_{1}, c_{2}$ and $c_{3}$ of the parity conserving (PC) Lagrangian, are taken into account. The TPV vertex generates also three body forces at order $Q^{0}$ while at order $Q^{1}$ all the time ordered diagrams cancel. The contribution of the contact terms appear at N2LO while the single nucleon contribution at LO. The final expression of the potential contains 11 LECs which must be determine from the experiment. The potential is then regularized introducing a regularization function $C_{\Lambda}(k)=\mathrm{e}^{-(k / \Lambda)^{4}}$. Three cutoff values are considered $\Lambda=450,500,550$ $\mathrm{MeV}$.

\section{The EDM of ${ }^{3} \mathrm{H}$ and ${ }^{3} \mathrm{He}$}

In this section we present the results for the EDM of the trinucleon system. The EDM of each nucleus can be expressed as the product of the LECs with numerical coefficients which contains all the dynamics given by the potential, namely,

$$
d^{A}=g_{0} a_{0}+g_{1} a_{1}+g_{2} a_{2}+\Delta a_{\Delta}+d_{p} a_{p}+d_{n} a_{n}+\sum_{i=1,5} C_{i} A_{i} .
$$


where the $C_{i}$ are the LECs which comes from the contact terms. In the calculation of the coefficients we use the various chiral order of the NN PC potential of Ref. [19] and for the three-nucleon PC potential the N2LO with the $c_{E}$ and $c_{D}$ LECs fitted in Ref. [20]. We evaluate also the theoretical errors associated with the chiral expansion truncation of the nuclear potential. We express the error on the numerical coefficients for the trinucleon systems as,

$$
\left(\delta a_{i}\right)^{2}=\left(\delta a_{i}^{\mathrm{PC}}\right)^{2}+\left(\delta a_{1}^{\mathrm{TRV}}\right)^{2}+\left(\delta a_{i}^{\psi}\right)^{2}
$$

where $\delta a_{i}^{\mathrm{PC}}$ is the error associated to the chiral expansion of the PC potential and $\delta a_{i}^{\mathrm{TRV}}$ the error associated with the chiral expansion of the TRV potential. Both the contributions were evaluated following the prescriptions of Ref. [21] where as reference momentum in the calculation of the errors we used the mass of the pion. Moreover we we introduce an error related to the uncertainties on the wave function $\delta a_{i}^{\psi}$ which we estimated to be of the order of $\sim 1 \%$. It is straightforward to understand that the errors are dominated by the TRV part because we are using the N2LO potential for it and the N4LO potential for the PC part.

The results for some of the coefficients of ${ }^{3} \mathrm{H}$ are shown in Figure 2 while for the ${ }^{3} \mathrm{He}$ in Figure 3.
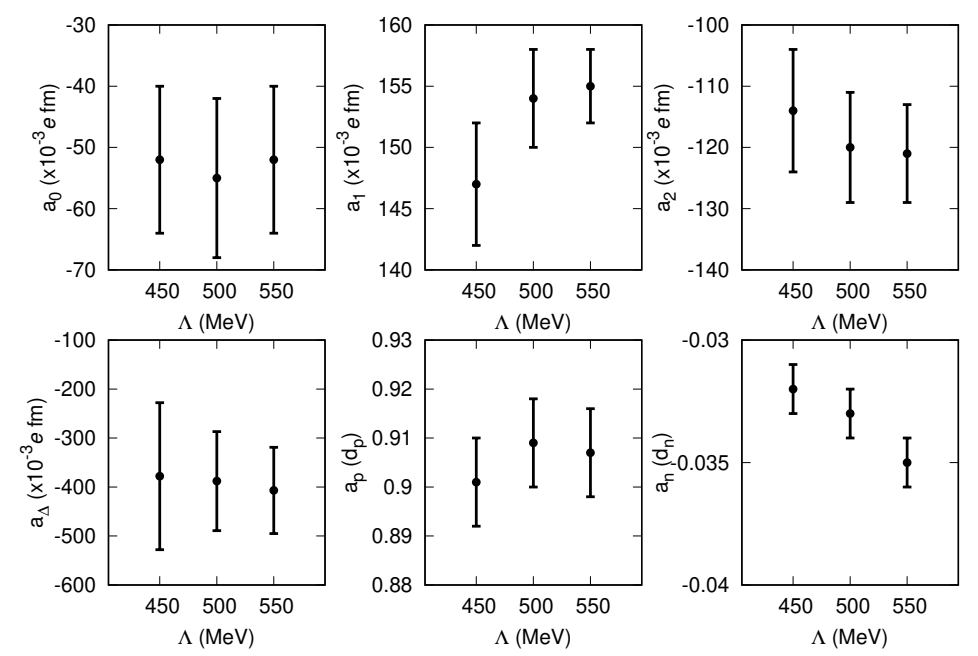

Figure 2: Values of selected coefficients $a_{i}$ for ${ }^{3} \mathrm{H}$. The error bars are evaluated following Ref. [21].

From the Figures it is possible to observe that the dependence on the cut-off is small, since all the results for a given $a_{i}$ are compatible within the theorethical uncertainties. Note the huge errors reported for the coefficients $a_{\Delta}$ which are due to the fact that the N2LO diagrams give a correction of $\sim 70 \%$, quite far away from what predicted by chiral perturbation theory. This effect is mainly due to the large contribution of the diagrams in which the $c_{1}, c_{2}$, and $c_{3}$ LECs [17] of the PC potential appear. We found that the TRV 3-body forces enhanches of the order of $\sim 25 \%$ the pure two-body contribution at NLO. This is an order of magnitude larger than the result reported in Ref. [22] however the discrepancy is not yet understood. The contribution of the TPE diagrams to $a_{0}$ and $a_{2}$ are of the order of $\sim 45 \%$ and $\sim 40 \%$ respectively which is larger than expected from 

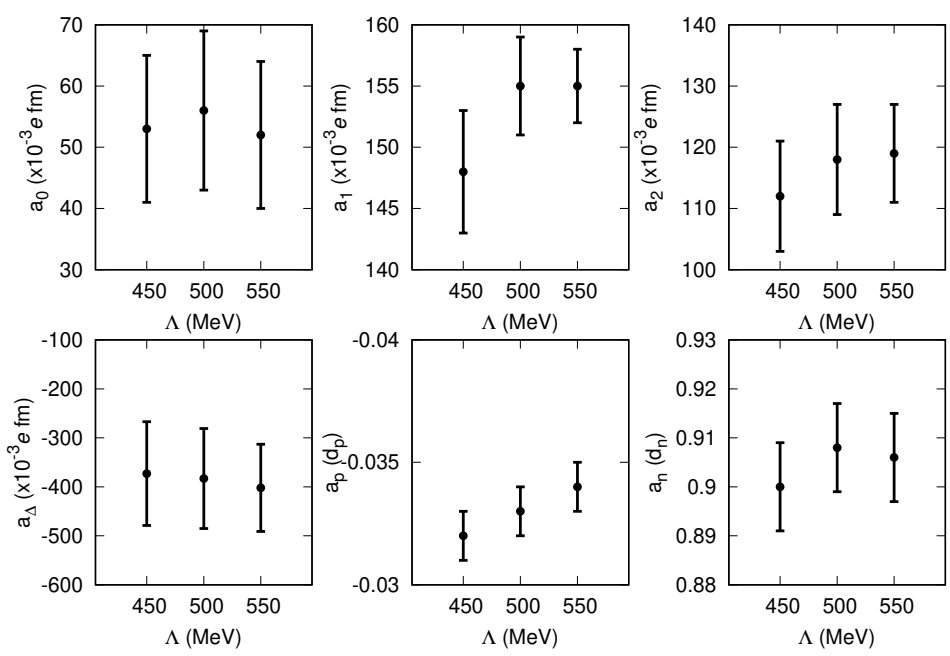

Figure 3: Values of selected coefficients $a_{i}$ for ${ }^{3} \mathrm{He}$. The error bars are evaluated following Ref. [21].

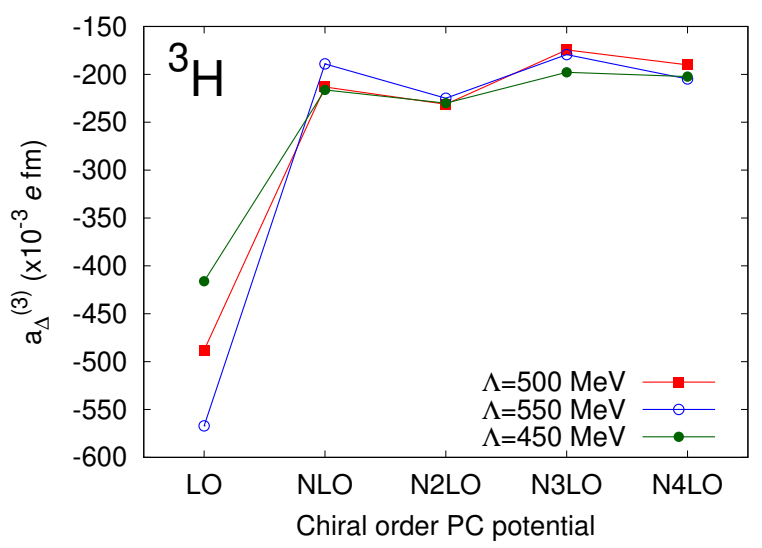

Figure 4: Values of the TRV 3-body contribution to $a_{\Delta}$ for the ${ }^{3} \mathrm{H}$ nucleus and for the three choice of the cutoff when varying the chiral order of the PC potential.

the chiral convergence and they are due mainly to the box diagrams of Figure 1. The errors of the $a_{1}$ coefficients results to be smaller compare to $a_{0}$ and $a_{2}$ because only relativistic corrections give contribution at N2LO. As reguarding $a_{p}$ and $a_{n}$ the error are mainly due to the uncertainties on the wave functions.

Moreover, we studied the effect of the PC potential on the EDM and we observe that the values evaluated with the N2LO, N3LO and N4LO PC potential differ by less than 5\% which confirm the robustness of the calculation. As example, in Figure 4 we report the value of the contribution of the TRV three body forces to $a_{\Delta}$ coefficient calculated with different chiral order of the PC potential and for the three different choices of the cutoff. 


\section{References}

[1] A. D. Sakharov, Violation of CP Invariance, C asymmetry, and baryon asymmetry of the universe, Pisma Zh. Eksp. Teor. Fiz. 5 (1967) 32.

[2] A. G. Cohen, D. B. Kaplan and A. E. Nelson, Progress in electroweak baryogenesis, Annual Review of Nuclear and Particle Science 43 (1993) 27 [https://doi.org/10.1146/annurev.ns.43.120193.000331].

[3] G. 't Hooft, Symmetry breaking through bell-jackiw anomalies, Phys. Rev. Lett. 37 (1976) 8.

[4] M. Pospelov and A. Ritz, Electric dipole moments as probes of new physics, Annals Phys. 318 (2005) 119 [hep-ph/0504231].

[5] C. A. Baker, D. D. Doyle, P. Geltenbort, K. Green, M. G. D. van der Grinten, P. G. Harris et al., Improved experimental limit on the electric dipole moment of the neutron, Phys. Rev. Lett. 97 (2006) 131801.

[6] W. C. Griffith, M. D. Swallows, T. H. Loftus, M. V. Romalis, B. R. Heckel and E. N. Fortson, Improved limit on the permanent electric dipole moment of ${ }^{199} \mathrm{Hg}$, Phys. Rev. Lett. 102 (2009) 101601.

[7] V. F. Dmitriev and R. A. Sen'kov, Schiff moment of the mercury nucleus and the proton dipole moment, Phys. Rev. Lett. 91 (2003) 212303.

[8] ACME collaboration, Order of Magnitude Smaller Limit on the Electric Dipole Moment of the Electron, Science 343 (2014) 269 [1310 . 7534].

[9] Y. F. Orlov, W. M. Morse and Y. K. Semertzidis, Resonance method of electric-dipole-moment measurements in storage rings, Phys. Rev. Lett. 96 (2006) 214802.

[10] Y. K. Semertzidis, A storage ring proton electric dipole moment experiment: most sensitive experiment to cp-violation beyond the standard model, Proceedings of the DPF 2011 Conference (2011) .

[11] A. Lehrach, B. Lorentz, W. Morse, N. Nikolaev and F. Rathmann, Precursor Experiments to Search for Permanent Electric Dipole Moments (EDMs) of Protons and Deuterons at COSY, 1201.5773.

[12] J. Pretz and on behalf of the JEDI collaboration, Measurement of permanent electric dipole moments of charged hadrons in storage rings, Hyperfine Interactions 214 (2013) 111.

[13] JEDI, SREDM collaboration, Search for electric dipole moments of light ions in storage rings, Phys. Part. Nucl. 45 (2014) 229.

[14] E. Mereghetti, W. H. Hockings and U. van Kolck, The Effective Chiral Lagrangian From the Theta Term, Annals Phys. 325 (2010) 2363 [1002.2391].

[15] J. de Vries, E. Mereghetti, R. Timmermans and U. van Kolck, The effective chiral lagrangian from dimension-six parity and time-reversal violation, Annals of Physics 338 (2013) 50 .

[16] J. Bsaisou, U.-G. Meissner, A. Nogga and A. Wirzba, P-and T-Violating Lagrangians in Chiral Effective Field Theory and Nuclear Electric Dipole Moments, Annals Phys. 359 (2015) 317 [1412.5471].

[17] A. Gnech and M. Viviani, Time Reversal Violation in Light Nuclei, 1906.09021.

[18] C. Maekawa, E. Mereghetti, J. de Vries and U. van Kolck, The time-reversal-and parity-violating nuclear potential in chiral effective theory, Nuclear Physics A 872 (2011) 117. 
[19] D. R. Entem, N. Kaiser, R. Machleidt and Y. Nosyk, Peripheral nucleon-nucleon scattering at fifth order of chiral perturbation theory, Phys. Rev. C 91 (2015) 014002.

[20] L. E. Marcucci, F. Sammarruca, M. Viviani and R. Machleidt, Momentum distributions and short-range correlations in the deuteron and ${ }^{3}$ He with modern chiral potentials, Phys. Rev. C99 (2019) 034003 [1809.01849].

[21] E. Epelbaum, H. Krebs and U.-G. Meißner, Precision nucleon-nucleon potential at fifth order in the chiral expansion, Phys. Rev. Lett. 115 (2015) 122301.

[22] J. Bsaisou, J. de Vries, C. Hanhart, S. Liebig, U.-G. Meissner, D. Minossi et al., Nuclear Electric Dipole Moments in Chiral Effective Field Theory, JHEP 03 (2015) 104 [1411. 580 4]. 